\title{
Utilization of Capture-Mark-Recapture for the Study of Recruitment and Population Growth Rate
}

\author{
R. Pradel ${ }^{1}$ \\ Cooperative Fish and Wildlife Research Unit, University of Florida, \\ Gainesville, Florida 32611, U.S.A.
}

\begin{abstract}
SUMMARY
Capture-mark-recapture data has been extensively used for the study of survival. However, recruitment and population growth rate can be investigated as well. The study of recruitment is shown to be equivalent to the study of survival in reverse and can be carried out by inverting capture histories. The natural parameter in this approach -here called seniority probability-.. is, at each occasion, the probability of being previously in the population. An overall likelihood is then presented that describes the gains and losses to the population by means of survival and seniority probabilitics. This likelihood can be easily modified for the direct study of population growth rates between occasions.
\end{abstract}

\section{Introduction}

In capture-mark-recapture (CMR) experiments, animals are captured, marked, released, and recaptured many times by repeated sampling. The result is a set of capture histories, one per observed animal, informative on survival, recruitment, and the size of the population. The early models of Leslic (1952) and Darroch (1959), and the fully stochastic models of Jolly (1965) and Seber (1965) addressed all of those questions. Later, CMR analysis for open populations has turned largely toward the study of survival. The flexibility achieved, stemming from the paper of Cormack (1964), has significantly contributed to the advancement of population biology by allowing survival to be (1) compared between populations and groups in the population and (2) tested for the effects of environmental variables (Lebreton, Pradel, and Clobert, 1993). The biological questions about fecundity and accession to reproduction whether for fundamental or applied purposes would probably largely benefit from a similar flexibility in the analysis of recruitment. Unfortunately, the current general models are not well suited to these aims: In the Jolly-Seber model, recruitment and population size are modeled by means of discrete estimators, whereas the continuous parameters proposed by Cormack $(1985,1989)$ and Crosbie and Manly (1985) are not directly interpretable in biological terms. The gap has been partially filled by elaborating on survival models in particular situations such as the recruitment of breeders within the population of origin (Lebreton et al., 1990; Clobert et al., 1994). In comparison, the approach to the analysis of recruitment we propose (Section 3 ) is at once general (undifferentiated recruitment) and specific (survival is not part of the models), allowing casy examination of questions of types (1) and (2), earlier. The simultaneous use of the recruitment parameters and of survival parameters in a single likelihood leads then to the study of population growth rates (PGRs) (Section 4). An example illustrates some aspects of the modeling of recruitment and the ability to directly estimate PGRs (Section 5).

\section{Assumptions and Notation}

The sampling scheme considered throughout the paper is that of $s$ successive random samples, not necessarily equally spaced over time. Animals captured are individually marked or noted if already marked and immediately released or removed. No mark is lost or misread. There is no temporary

${ }^{1}$ Permanent address: C.E.F.E./C.N.R.S., BP 5051, 34033 Montpellier Cedex, France.

Key words: Constrained models; Jolly-Seber model; Likelihood; Open models; Populations dynamics; Population growth rate; Seniority probabilities; Survival. 
emigration (except possibly random). Animals are further supposed to be identical and uniquely identifiable and to have independent fates. Finally, for convenience, parameters are considered in the proofs as time-specific, although the approach proposed is valid more broadly (e.g., for constant survival and/or capture probabilities).

The following notation is used throughout.

$d=$ data set,

$h=$ capture history,

$\epsilon_{i}=$ cvent at time $i(0=$ nonobservation, $1=$ observation $)$,

$\eta=$ indicator of right censorship ( $\eta$ equals 1 if the animal was removed, 0 if it was released),

$e=$ index of the earliest observation in $h$,

$l=$ index of the last observation in $h$.

The notation \#\{conditions $\}$ hereafter designates the number of animals that fulfill the conditions.

$u_{i}=$ number of animals observed for the first time at $i(\#\{e=i\})$,

$n_{i}=$ number of animals observed at $i\left(\#\left\{\epsilon_{i}=1\right\}\right)$,

$v_{i}=$ number of animals observed for the last time at $i(\#\{l=i\})$,

$d_{i}=$ number of animals removed from the population at $i(\#\{l=i, \eta=1\})$.

"just before" and "just after" used in what follows for the sake of generality can be replaced with "at" when there are no removals nor introductions.

$\gamma_{i}=$ seniority probability, probability that an animal present just before time $i$ was already present just after time $i-1$,

$\phi_{i}=$ survival probability, probability that an animal present just after time $i$ will still be present just before time $i+1$,

$p_{i}\left(r_{i}\right)=$ capture probability, probability of being captured at time $i$ for an individual present just before (after) time $i$ ( $p_{i}$ and $r_{i}$ differ if some animals are removed),

$\rho_{i}=$ population growth rate between occasion $i$ and occasion $i+1$,

$\mu_{i}=$ probability of being released for an individual captured at time $i$,

$\xi_{i}=$ probability of not being seen before time $i$ for an animal present just before time $i$,

$\chi_{i}=$ probability of not being seen after time $i$ for an animal present just after time $i$,

$N_{i}=$ expected population size at time $i$ (we will distinguish $N_{i}^{-}$, the population size just before the sample $i$ is taken, from $N_{i}^{+}$, the population size just after the releases at time $i$ ).

\section{The Recruitment Analysis}

The idea here is to condition on the time of the last observation and to read the capture histories backward. This induces a simple "duality" relationship between survival and recruitment models in which the natural counterpart of the survival probability is the probability of being in the population earlier than the present date. This is the seniority probability of Section 2 and the inverse of the dilution rate in Jolly $(1965$, p. 234). For instance, with $s=6$, the capture history 010100 can be read: The animal was last caught at occasion 4 ; it was not recruited between occasion 4 and 3 , was not captured at 3, was not recruited between 3 and 2 , was captured at 2, and was not seen earlier than 2 . Thus, conditional on the last capture at 4 , its associated probability is $\gamma_{4}\left(1-r_{3}\right) \gamma_{3} r_{2} \xi_{2}$. Now, if we write the capture history backward, that is, as 001010 , the associated probability, conditional on the first capture at 3 , would be $\phi_{3}\left(1-p_{4}\right) \phi_{4} p_{5} \chi_{5}$. One can observe a formal correspondence between the two expressions.

More generally, the probability of the generic capture history $h=\epsilon_{1}, \ldots, \epsilon_{s}$ in the recruitment analysis is

$$
P_{r}(h)=\left\{\prod_{i=\epsilon+1}^{l} \gamma_{i}\right\}\left\{\prod_{i=e}^{l-1} r_{i}^{\epsilon_{i}}\left(1-r_{i}\right)^{1-\epsilon_{i}}\right\} \xi_{e},
$$

where $\xi_{i}=\left(1-\gamma_{i}\right)+\gamma_{i}\left(1-r_{i-1}\right) \xi_{i-1}, i=2, \ldots, s\left(\xi_{1}=1\right)$, whereas in the survival analysis, it is

$$
P_{s}(h)=\left\{\prod_{i=\ell}^{l-1} \phi_{i}\right\}\left\{\prod_{i=e+1}^{l} p_{i}^{\epsilon_{i}}\left(1-p_{i}\right)^{1-c_{i}}\right\} \chi_{l},
$$

where $\chi_{i}=\left(1-\phi_{i}\right)+\phi_{i}\left(1-p_{i+1}\right) \chi_{i+1}, i=1, \ldots, s-1\left(\chi_{\mathrm{s}}=1\right)$. Reversing $h$ anounts to renumbering the occasions from the last one (change of index $j=s-i+1$ ). The second expression, 
applied to the reverse of $h$, can then easily be shown to be formally equivalent to the first with $\phi_{1}, \phi_{2}, \ldots, \phi_{s-1}$ corresponding, respectively, to $\gamma_{s}, \gamma_{s-1}, \ldots, \gamma_{2}$ and $p_{2}, p_{3}, \ldots, p_{s}$ corresponding, respectively, to $r_{s-1}, r_{s-2}, \ldots, r_{1}$. The recruitment analysis of a data set $d$ is thus identical to the survival analysis of its dual (reverse) data set $d^{\prime}$.

In practice, carrying out a survival analysis of the reverse data set to study recruitment will work with all models nested in the model-denoted $\left(\gamma_{t * g}, r_{t * g}\right)$ by analogy with the survival models notation (Lcbreton et al., 1992)-where seniority and capture probabilities vary over time independently in $g$ groups. This includes, among others, models with parameters common to several groups and models with parameters constant over time or functions of an external variable. Two important limitations of the approach are trap and age (counted from marking time) dependence, which obviously depend on the direction of time.

\section{The Overall or PGR Analysis in the Time-Specific Context}

We reason here conditionally on the total number captured. The estimation of recruitment and survival parameters is then based on the multinotuial distribution of the ever seen animals among the potential capture histories (independent and identical individuals).

Moreover, relations among population sizes at different occasions follow from conditional binomial distributions that result from the hypotheses of Section 2. Thus, the expected number of animals present both just after $i$ and just before $i+1$ is, from the survival point of view, $N_{i}^{+} \phi_{i}$ and, from the recruitment point of view, $N_{i+1}^{-} \gamma_{i+1}$. Hence,

$$
\rho_{i}=\frac{N_{i+1}^{-}}{N_{i}^{+}}=\frac{\phi_{i}}{\gamma_{i+1}}
$$

$N_{i}^{-}$and $N_{i}^{+}$differ if some animals are removed: $N_{i}^{+}=N_{i}^{-}\left(1-p_{i}\right)+N_{i}^{-} p_{i} \mu_{i}=N_{i}^{-}\left(1-p_{i}\left(1-\mu_{i}\right)\right)$ so that

$$
\frac{N_{i+1}^{-}}{N_{i}^{-}}=\rho_{i} \frac{N_{i}^{+}}{N_{i}^{-}}=\frac{\phi_{i}\left(1-p_{i}\left(1-\mu_{i}\right)\right)}{\gamma_{i+1}} .
$$

Finally, for the same reason, the probability of being xnissed at occasion $i$ for an animal present in the population just after $i$ is $1-r_{i}=\left(1-p_{i}\right) /\left(1-p_{i}\left(1-\mu_{i}\right)\right)$. Consequently,

$$
\xi_{i}=\left(1-\gamma_{i}\right)+\gamma_{i} \frac{1-p_{i-1}}{1-p_{i-1}\left(1-\mu_{i-1}\right)} \xi_{i-1}, \quad i=2, \ldots, s\left(\xi_{1}=1\right) .
$$

The probability of each capture history $h$ conditional on the total number captured can now be reached as the ratio of the expected number of animals, $m_{h}$, with this capture history to the expected total number of animals captured.

$$
m_{h}=N_{e}^{-} \xi_{e} p_{e} \mu_{e} P_{s}(h),
$$

where

$$
P_{s}(h)=\left\{\prod_{i=e}^{l-1} \phi_{i}\right\}\left\{\prod_{i=\epsilon_{+}+1}^{l-1}\left(p_{i} \mu_{i}\right)^{\epsilon_{i}}\left(1-p_{i}\right)^{1-\epsilon_{i}}\right\} p_{l}\left(1-\mu_{l}\right)^{\eta}\left(\mu_{l} \chi_{l}\right)^{1-\eta}
$$

( $P_{s}(h)$ is the usual probability of $h$ in the survival model except that the removal process is modeled.) By noting that

$$
N_{e}^{-}=N_{1}\left(\prod_{i=1}^{e-1} \frac{N_{i+1}^{-}}{N_{i}^{-}}\right)=N_{1} \frac{\prod_{i=1}^{i-1} \phi_{i}\left(1-p_{i}\left(1-\mu_{i}\right)\right)}{\prod_{i=2}^{e} \gamma_{i}}
$$


$m_{h}$ can be written

$$
m_{h}=N_{1}^{-} \prod_{\frac{\xi_{i}}{\xi_{i=1}}}^{\prod_{e}^{e} \gamma_{i}}\left\{\prod_{i=e}^{l} p_{i}^{\epsilon_{i}}\left(1-p_{i}\right)^{1-\epsilon_{i}}\right\}\left\{\prod_{i=e}^{l-1} \mu_{i}^{\epsilon_{i}}\right\}\left\{\prod_{i=1}^{e-1}\left(1-p_{i}\left(1-\mu_{i}\right)\right)\right\}\left(1-\mu_{l}\right)^{\eta}\left(\mu_{l} \chi_{l}\right)^{1-\eta} .
$$

The sum $M$ of the $m_{h}$ 's over all observable capture histories can be conveniently calculated as the sum of the expected number of animals seen for the first time at each occasion,

$$
M=\sum_{i=1}^{s} \xi_{i} N_{i}^{-} p_{i}=N_{1}^{-} \sum_{i=1}^{s} \xi_{i} \frac{\prod_{j=1}^{i-1} \phi_{j}\left(1-p_{j}\left(1-\mu_{j}\right)\right)}{\prod_{j=2}^{i} \gamma_{j}} p_{i} .
$$

The conditional probability of $h, P_{g}(h)=m_{h} / M$ is therefore

$$
\begin{aligned}
P_{g}(h)= & \xi_{e}\left\{\prod_{i=e+1}^{s} \gamma_{i}\right\}\left\{\prod_{i=e}^{l} p_{i}^{\epsilon_{i}}\left(1-p_{i}\right)^{1-\epsilon_{i}}\right\}\left\{\prod_{i=1}^{l-1} \phi_{i}\right\}\left\{\prod_{i=e}^{l-1} \mu_{i}^{\epsilon_{i}}\right\}\left\{\prod_{i=1}^{e-1}\left(1-p_{i}\left(1-\mu_{i}\right)\right)\right\} \\
& \cdot\left(1-\mu_{l}\right)^{\eta}\left(\mu_{l} \chi_{l}\right)^{1-\eta} / \sum_{i=1}^{s} \xi_{i}\left\{\prod_{j=1}^{i-1} \phi_{j}\left(1-p_{j}\left(1-\mu_{j}\right)\right)\right\}\left\{\prod_{j=i+1}^{s} \gamma_{j}\right\} p_{i} .
\end{aligned}
$$

The likelihood $L$ for a set $\Omega$ of observed animals $\omega$ is the product of the conditional probabilities of all individual capture histories.

$$
L=\prod_{\omega} P_{g}(h(\omega))=\prod_{h} P_{g}(h)^{\#\{h\}} .
$$

In terms of the $u_{i}$ 's, $n_{i}$ 's, $v_{i}$ 's, and $d_{i}$ 's,

$$
\begin{aligned}
& L(\phi, p, \gamma, \mu)=\prod_{i=1}^{s}\left(\xi_{i}^{u_{i}}\right)\left(\gamma_{i}^{\sum^{\sum<i} u_{j}}\right)\left(p_{i}^{n_{i}}\right)\left[\left(1-p_{i}\right)^{\sum_{j \leq i} u_{j}-\sum_{j<i} v_{j}-n_{i}}\right]\left(\phi_{i}{ }^{\sum_{j i} v_{j}}\right)\left(\mu_{i}{ }^{n_{i}-d_{i}}\right) \\
& \cdot\left[\left(1-\mu_{i}\right)^{d_{i}}\right]\left[\left(1-p_{i}\left(1-\mu_{i}\right)\right)^{\sum_{j>i}^{u_{j}}}\right]\left(\chi_{i}^{{ }^{v_{i}-d_{i}}}\right) \\
& /\left(\sum_{i=1}^{s} \xi_{i}\left\{\prod_{j=1}^{i-1} \phi_{j}\left(1-p_{j}\left(1-\mu_{j}\right)\right)\right\}\left\{\prod_{j=i+1}^{s} \gamma_{j}\right\} p_{i}\right)^{\sum_{i=1}^{s} u_{i}} \text {. }
\end{aligned}
$$

\section{Example}

The previous results are now applied to the classical example of a population of female black-kneed capsids (Jolly, 1965). A set of sufficient statistics for the Cormack Jolly-Seber model (Cormack, 1964; Jolly, 1965; Seber, 1965) comprises the numbers released at each occasion ( $s_{t}$ in Jolly's notation) and the numbers among those released at $j$ that are next recaptured at $i$ ( $n_{i j}$ in Jolly's notation). The same quantities from the dual data set are, respectively, the numbers captured at each occasion ( $n_{t}$ in Jolly's notation) and the numbers among those captured at $j$ that were last released at $i$ (again the $n_{i j}$ 's). The goodness-of-fit test based on those quantities (Burnham et al., 1987) (and incomplete as such because the individual capture histories for this data set are not available) leads to acceptance of model $\left(\gamma_{t}, r_{t}\right)\left(\chi^{2}(44)=50.49, P=0.77\right)$ as a starting point for recruitment analysis.

Estimates of the proportions of new recruits (and their confidence intervals) are then obtained numerically using the program SURGE (Pradel and Lebreton, 1991) under different models (Table 1). Time dependency and constancy over time were considered for seniority and capture probabilities and, for the seniority probabilities only, two types of trends over time: logistic linear and 
logistic quadratic lincar. The best model was selected on the basis of minimum Akaike information criterion. All this is standard methodology in survival analysis (Lebreton et al., 1992). The constancy of capture probabilities was accepted (likelihood ratio test [LRT] between models $\left(\gamma_{t}, r_{t}\right)$ and $\left(\gamma_{t}, r\right): \chi^{2}(10)=7.32, P=0.70$ ), but the constancy of $\gamma$ was then strongly rejected (LRT between $\left(\gamma_{t}, r\right)$ and $\left.(\gamma, r): \chi^{2}(11)=186.98, P<10^{-5}\right)$. A model with a trend in $\gamma$ of the form $\operatorname{logit}(\gamma)=a+b T$ (Clobert and Lebreton, 1985) yielded an apparently significant positive slope $\left(b=0.274, \sigma_{b}=0.026\right)$, that is, emergences and immigration declined; however, this specific form of variation over time was insufficient to model the time dependence in $\gamma\left(\right.$ LRT between $\left(\gamma_{t}, r\right)$ and $\left.\left(\gamma_{a+b T}, r\right): \chi^{2}(10)=41.44, P<10^{-4}\right)$. The second-order trend was not accepted either (LRT between $\left(\gamma_{t}, r\right)$ and $\left.\left(\gamma_{a+b T+c^{2}}, r\right): \chi^{2}(9)=32.66, P<10^{-3}\right)$. Table 2 gives the maximum likelihood estimates of the $\gamma$ parameters under the best model $\left(\gamma_{t}, r\right)$.

Table 1

Analysis of recruitment of female black-kneed capsid. The multinomial models of capture-recapture are designated by the structure put on the parameters; for instance, $\gamma_{a+b T}, r$ means linear trend on the seniority probabilities (on a logit scale), capture rate constant. Then, follow the number of identifiable parameters, the relative deviance of the model (as given by program SURGE), and the Akaike information criterion (AIC) for comparison between models $(A I C=$ deviance $+2 * \#$ par $)$.

\begin{tabular}{lccc}
\hline \multicolumn{1}{c}{ Model } & \# par & Deviance & AIC \\
\hline$\gamma_{t}, r_{t}$ & 23 & 4896.11 & 4942.11 \\
$\gamma_{t}, r$ & 13 & 4903.43 & 4929.43 \\
$\gamma_{1} r$ & 2 & 5090.41 & 5094.41 \\
$\gamma_{a+b T}, r$ & 3 & 4944.87 & 4950.87 \\
$\gamma_{a+b T^{\prime}+c T^{2}, r}$ & 4 & 4936.09 & 4944.09 \\
\hline
\end{tabular}

Table 2

Estimates of seniority probabilities $\gamma$ for a population of female black-kneed capsids when $r$ is constant. Confidence intervals are based on the normal asymptotic distribution of the estimators; they cannot be computed for parameters estimated on a boundary. $\gamma_{2}$ is not estimable.

\begin{tabular}{rccc}
\hline \hline & & \multicolumn{2}{c}{$95 \%$ Confidence interval } \\
\cline { 3 - 4 }$i$ & $\tilde{\gamma}_{i}$ & Lower & Upper \\
\hline 3 & 0.794 & 0.584 & 0.914 \\
4 & 0.666 & 0.529 & 0.779 \\
5 & 0.544 & 0.443 & 0.641 \\
6 & 0.855 & 0.663 & 0.947 \\
7 & 0.789 & 0.638 & 0.885 \\
8 & 0.858 & 0.714 & 0.936 \\
9 & 1.000 & - & 0.979 \\
10 & 0.918 & 0.725 & 0.940 \\
11 & 0.850 & 0.672 & 0.918 \\
12 & 0.824 & 0.663 & 0.998 \\
13 & 0.955 & 0.502 & \\
\hline
\end{tabular}

For the simultaneous study of recruitment and death processes, likelihood (2) was maximized using a quasi-Newton algorithm (implemented in language Gauss). The likelihood was successively treated as a function of the $\phi$ and $\gamma$ parameters constrained within [0,1] by a logistic transform and as a function of the $\phi$ and $\rho$ parameters (substituting $\gamma$ from (1)) with the $\phi$ 's constrained again within $[0,1]$ by a logistic transform and the $\rho$ 's forced to remain positive by a logarithmic transform. This second set of conditions is, in fact, less strict, as it allows values of $\gamma$ (computable from $\phi$ and $\rho$ using (1)) greater than 1 . As a consequence, the minimum reached differed slightly in the two cases (Tables $3 a$ and $3 b$ ). Also, convergence was difficult to achieve in the second case. The control over convergence and over the reasonableness of the estimates was thus weaker with $\rho$ as a fundamental parameter.

For both types of analyses, with no constraint applied and time-dependent parameters, the estimates were exactly those of Jolly (1965). 


\section{Table 3}

Maximum likelihood estimates and $95 \%$ confidence intervals for a population of female black-kneed capsids under the overall model: numerical optimization of $(a) L(\phi, p, \gamma, \mu)$ and $(b) L(\phi, p, \rho, \mu)$ where $L$ is the logarithmic likelihood. Confidence intervals are based on the normal asymptotic distribution of the estimators; they cannot be computed for parameters estimated on a boundary. $\hat{\rho}_{i}$ (case (a)) and $\hat{\gamma}_{i}$ (case (b)) are computed, respectively, as $\hat{\phi}_{i} / \hat{\gamma}_{i+1}$ and $\hat{\phi}_{i-1} / \hat{\rho}_{i-1}$ for the sake of comparison. ${ }^{*}$

\begin{tabular}{|c|c|c|c|c|c|}
\hline \multicolumn{6}{|c|}{ (a) $\phi$ and $\gamma$ parametrization } \\
\hline$i$ & $\hat{\phi}_{i}$ & $\hat{p}_{i}$ & $\hat{\gamma}_{i}$ & $\hat{\mu}_{i}$ & $\hat{\rho}_{i}$ \\
\hline 1 & $0.65(0.44 \quad 0.82)$ & & & & \\
\hline 2 & $1.00(\mathrm{NA})$ & $0.28(0.15-0.47)$ & & $0.98(0.94-0.99)$ & 1.49 \\
\hline 3 & $0.87(0.57-0.97)$ & $0.22(0.17-0.28)$ & $0.67(0.25-0.92)$ & $0.97(0.93-0.99)$ & 1.26 \\
\hline 4 & $0.56(0.45-0.68)$ & $0.22(0.16 \cdots 0.29)$ & $0.69(0.46-0.86)$ & $0.97(0.93-0.98)$ & 0.98 \\
\hline 5 & $0.84(0.65-0.93)$ & $0.23(0.17-0.30)$ & $0.57(0.39-0.73)$ & $0.97(0.94-0.99)$ & 0.94 \\
\hline 6 & $0.79\left(\begin{array}{lll}0.62 & 0.90)\end{array}\right.$ & $0.24(0.19-0.30)$ & $0.89\left(\begin{array}{lll}0.43 & 0.99)\end{array}\right.$ & $0.99(0.96-1.00)$ & 0.91 \\
\hline 7 & $0.65(0.53-0.75)$ & $0.31(0.25 \cdot 0.38)$ & $0.87(0.59-0.97)$ & $0.97(0.94-0.99)$ & 0.76 \\
\hline 8 & $0.99(0.00-1.00)$ & $0.27(0.23-0.33)$ & $0.80(0.65-0.90)$ & $0.99(0.96-1.00)$ & 0.99 \\
\hline 9 & $0.69(0.52 \cdot 0.82)$ & $0.27(0.22-0.33)$ & $1.00(\mathrm{NA})$ & $0.98(0.95-0.99)$ & 0.77 \\
\hline 10 & $0.88(0.54-0.98)$ & $0.27\left(\begin{array}{lll}0.21 & 0.34)\end{array}\right.$ & $0.90(0.69-0.98)$ & $0.99(0.95-1.00)$ & 1.06 \\
\hline 11 & $0.77(0.47-0.93)$ & $0.24(0.18 \quad 0.31)$ & $0.83(0.65-0.93)$ & $0.98\left(\begin{array}{lll}0.93 & 0.99)\end{array}\right.$ & 0.92 \\
\hline 12 & & $0.26(0.18-0.35)$ & $0.84(0.63-0.94)$ & $1.00(\mathrm{NA})$ & \\
\hline 13 & & & $0.95(0.45 \cdot 1.00)$ & & \\
\hline \multicolumn{6}{|c|}{ (b) $\phi$ and $\rho$ parametrization } \\
\hline$i$ & $\hat{\phi}_{i}$ & $\hat{p}_{i}$ & $\hat{\rho}_{i}$ & $\hat{\mu}_{i}$ & $\hat{\gamma}_{i}$ \\
\hline 1 & $0.65\left(\begin{array}{lll}0.41 & 0.83)\end{array}\right.$ & & & & \\
\hline 2 & $1.00(\mathrm{NA})$ & $0.28\left(\begin{array}{lll}0.16 & 0.46\end{array}\right)$ & $1.50(0.84-2.70)$ & $0.98\left(\begin{array}{lll}0.94 & 0.99\end{array}\right)$ & \\
\hline 3 & $0.87(0.61-0.97)$ & $0.22(0.16-0.29)$ & $1.26(0.88-1.82)$ & $0.97(0.93-0.99)$ & 0.66 \\
\hline 4 & $0.56\left(\begin{array}{ll}0.45 & 0.67)\end{array}\right.$ & $0.22(0.16-0.29)$ & $0.99\left(\begin{array}{lll}0.69 & 1.41)\end{array}\right.$ & $0.97(0.93-0.98)$ & 0.69 \\
\hline 5 & $0.84(0.65-0.93)$ & $0.23(0.17-0.30)$ & $0.94(0.69-1.29)$ & $0.97\left(\begin{array}{lll}0.94 & 0.99)\end{array}\right.$ & 0.57 \\
\hline 6 & $0.79(0.63-0.89)$ & $0.24(0.19-0.30)$ & $0.91(0.70-1.18)$ & $0.99(0.96-1.00)$ & 0.89 \\
\hline 7 & $0.65(0.54-0.75)$ & $0.31(0.25-0.38)$ & $0.82(0.64-1.05)$ & $0.97(0.94-0.99)$ & 0.87 \\
\hline 8 & $0.98(0.00-1.00)$ & $0.27(0.21-0.33)$ & $0.96(0.77-1.20)$ & $0.99(0.96-1.00)$ & 0.7 .9 \\
\hline 9 & $0.69(0.52-0.81)$ & $0.27(0.22-0.33)$ & $0.76(0.58-1.01)$ & $0.98\left(\begin{array}{lll}0.95 & 0.99\end{array}\right)$ & 1.02 \\
\hline 10 & $0.88(0.51-0.98)$ & $0.27(0.20-0.34)$ & $1.06(0.79-1.43)$ & $0.99(0.95-1.00)$ & 0.91 \\
\hline 11 & $0.77(0.49-0.92)$ & $0.24(0.18-0.31)$ & $0.92(0.66-1.29)$ & $0.98(0.93-0.99)$ & 0.83 \\
\hline 12 & & $0.26\left(\begin{array}{lll}0.19 & 0.34\end{array}\right)$ & & $1.00(\mathrm{NA})$ & 0.84 \\
\hline 13 & & & & & \\
\hline
\end{tabular}

a NA, not available.

\section{Discussion}

The approach proposed is an attempt to introduce more flexibility in the analysis of CMR data beyond the study of survival. The interest of the present parametrization as compared to those of Jolly (1965) or Cormack (1989) lies in the possibility of manipulating directly and, hence, testing against variables, such quantities as fecundity rates and population growth rates. This may necessitate replacing the seniority probabilities in likelihood expression (2); for instance, to directly study fecundity rates $f_{i}$, one may substitute $1 /\left(1+f_{i}\right)$ for $\gamma_{i}$ (population without immigration). Section 5 has demonstrated that, in practice, recruitment, in the form of seniority probabilities, can be estimated and tested for trend and that PGRs can be estimated as well. The possibility to actually test those two types of parameters against variables and among groups is also clearly at hand (although numerical problems may have to be solved).

There is currently no specialized software for the global approach, and this is a transitory reason for using the partial analyses for which SURGE (Pradel and Lebreton, 1991) and similar software can be used. Another more fundamental reason is that the conditioning in the partial analyses protects against structural bias; overlooking age specificity in survival, for example, could affect recruitment estimates. Therefore, if one is interested in recruitment and has limited information on survival, or if the survival structure is complex, it might be better to use the recruitment-only approach. 


\section{ACKNOWLEDGEMENTS}

This work, begun under the supervision of J.-D. Lebreton, was boosted by discussions with J. D. Nichols on the necessity to introduce fecundity into the likelihood for CMR data. It was funded by the Patuxent Wildlife Research Center of the U.S. Fish and Wildlife Service. Many thanks are due to J. Hines for implementing the optimization of the global likelihood in language GAUSS. J.-D. Lebreton, J. D. Nichols, F. Cooke, P. O'Hara, and anonymous referees helped improve an earlier version.

\section{RÉSUMÉ}

Lues données de capture-marquage-recapture ont été trc̀s largenent utilisées pour étudier la survie. Elles peuvent cependant aussi servir à l'étude du recrutement et du taux de croissance des populations. On montre ici que l'étude du recrutement est équivalent à une étude de survie menée sur les histoires de capture renversées. Le paramètre naturel dans cette approche appelé probabilité d'ancienneté est, à chaque occasion, la probabilité d'avoir préalablement appartenu à la population. Une vraisemblance générale est ensuite donnée dans laquelle gains et pertes de la population sont décrits à l'aide des probabilités de survic et d'ancienneté. Cette vraiscmblance pent facilement être modifiée pour faire apparaitre le taux de croissance de la population d'une occasion sur l'autre.

\section{REFHRENCES}

Burnham K. P., Anderson D. R., White G. C., Brownie C., and Pollock, K. H. (1987). Design and analysis methods for fish survival experiments based on release-recapture. American Fisheries Society Monograph 5, 437 pp.

Clobert, J. and Lebreton, J.-D. (1985). Dépendance de facteurs de milieu dans les estimations de taux de survie par capture-recapture. Biometrics 41, 1031-1037.

Clobert, J., Lebreton, J.-D., Allainé, D., and Gaillard, J.-M. (1994). The estimation of age-specific breeding probabilities from recaptures or resightings in vertebrate populations II. Longitudinal models. Biometrics 50, $375 \cdot 385$.

Cormack, R. M. (1964). Estimates of survival from the sighting of marked animals. Biometrika 51, $429-438$.

Cormack, R. M. (1985). Examples of the use of GLIM to analyse capture-recapture studies. In Statistics in Ornithology, P. M. North and B. J. T. Morgan (eds), 243 273. Berlin: SpringerVerlag.

Cormack, R. M. (1989). Loglinear models for capture-recapture. Biometrics 45, 395-413.

Crosbie, S. F. and Manly, B. F. J. (1985). Parsimonious modelling of capture-mark-recapture studies. Biometrics 41, 385-398.

Darroch, J. N. (1959). The multiple-recapture census. II. Estimation when there is immigration or death. Biometrika 46, 336-351.

Jolly, G. M. (1965). Explicit estimates from capture-recapture data with both death and immigration-stochastic models. Biometrika 52, 225-247.

Lebreton, J.-D., Burnham, K. P., Clobert, J., and Anderson, D. R. (1992). Modeling survival and testing biological hypotheses using marked animals: A unified approach with case studies. Ecological Monographs 62, 67-118.

Lebreton, J. D., Hemery, G., Clobert, J., and Coquillard, H. (1990). The estimation of age-specific brecding probabilities from recaptures or resightings in vertebrate populations I. Transversal models. Biometrics 46, 127-140.

Lebreton, J. D., Pradel, R., and Clobert, J. (1993). The statistical analysis of survival in animal populations. Trends in Ecology and Evolution 8, 91-95.

Leslie, P. H. (1952). The estimation of population parameters obtained by means of the capturerecapture method. II. The estimation of total numbers. Biometrika 39, 363388.

Pradel, R. and Lebreton, J. D. (1991). User's Manual for Program SURGE, version 4.1. Montpellier, France: C.E.P.E./C.N.R.S.

Seber, G. A. F. (1965). A note on the multiple recapture census. Biometrika 52, 249259. 